Journal of Advanced College of Engineering and Management, Vol. 6, 2021

\title{
ASSESSMENT OF RELATIONSHIP BETWEEN ROAD ROUGHNESS AND PAVEMENT SURFACE CONDITION
}

\author{
Satkar Shrestha ${ }^{1}$, Rajesh Khadka ${ }^{2}$ \\ ${ }^{1}$ Fusion Engineering Services, Lalitpur, Nepal; satkarshrestha@gmail.com \\ ${ }^{2}$ Assistant Professor, Department of Transportation Engineering, Nepal Engineering College, PU.
}

\begin{abstract}
Pavement evaluation is the most significant procedure to minimize the degradation of the pavement both functionally and structurally. Proper evaluation of pavement is hence required to prolong the life year of the pavement, which thus needs to be addressed in the policy level. By this, the development of genuine indices are to be formulated and used for the evaluation. In context of evaluating the pavement indices for measuring the pavement roughness, International Roughness Index (IRI) is used, whereas for calculating the surface distress, indices as such Surface Distress Index (SDI) and Pavement Condition Index (PCI) are used. Past evaluating schemes used by Department of Roads (DOR) were limited to IRI for evaluating the pavement roughness and SDI for measuring the surface distress, which has least variability in categorizing the pavement according to the deformation. Apart from these, PCI which has wide range of categories for evaluating pavement, is not seen in practice in Nepal due to its cumbersome field work and calculations. In this paper the relationship is developed relating PCI with IRI and SDI using regression analysis by using Microsoft excel. In the other words, the pavement roughness index is compared with the surface distress indices. In 2017, 23.6Km of feeder roads in various locations of Kathmandu and Lalitpur districts were taken for this study which comprised of 236 sample data, each segmented to $100 \mathrm{~m}$. For this, IRI was sourced as secondary data, obtained from Highway Maintenance and Information System (HMIS) unit, Kathmandu, whereas, PCI and SDI were calculated from the field data obtained from the survey carried out in those sections manually. Then after, among 236 samples, 189 samples were taken for the relationship development which was then validated using 47 remaining samples. Furthermore, in the year, 2019 additional $3 \mathrm{Km}$ of data was taken for validating the obtained relationships. It was done to improve the numerical predictions of data with such variation and thus satisfactory relationships were developed among the indices discussed in this study. The regression relationships between the two indices, IRI-PCI and IRI-SDI were thus significantly obtained. It has been found that the $\mathrm{R}^{2}$ value for these relationships developed were statistically significant with $5 \%$ level of significance. The $\mathrm{R}^{2}$ value for all the relationships showed that these relationships could be used for predicting the indices which would help in evaluating the pavement.
\end{abstract}

Keywords: Pavement Evaluation; Pavement Evaluating Indices;International Roughness Index (IRI); Surface Distress Index (SDI); Pavement Condition Index (PCI); Department of Roads (DOR)

\section{Introduction}

The pavement deterioration is the process by which distress (defects) develops in the road pavement under the combined effects of traffic loading and environmental conditions [1]. For the effective maintenance and repair of these network systems, the evaluation of the pavement needs to be conducted in regular interval. The Department of Roads (DOR) of the Government of Nepal (GON) conducts pavement condition assessments each year to determine the physical status of the roadway network. The type of pavement improvement is dictated by the extent of the distress severity. There are various applicable indices for the measurement of distress and ride quality. Some measure the structural damages, whereas some measure, the functional adequacy. The functional adequacy can be evaluated using indices such as Pavement Condition Index (PCI), International Roughness Index (IRI) and Surface Distress Index (SDI). Pavement roughness is measured by various automatic multifunctional measuring instruments or devices and is quantified using the International Roughness Index (IRI), an internationally accepted parameter. The ride quality indicates the smoothness of the roadway segment which is reported as the International Roughness Index (IRI) [2]. 


\section{Literature Review}

The pavement distresses are those distresses which are visible on the pavement surface. The form and severity of distress a pavement has, can provide great insight into what its future maintenance and/or rehabilitation will be needed. The distress is generally described in term of severity, extent and distress type. Deterioration of the pavement is very complex, as it entails structural weakness and functional distress. This distressing phenomenon is explained by the interactions between traffic, climate, material, time and the geometric features of the roadway [3]. Defects in flexible pavement can be measured on the basis of appearance and unit of measurements carried are described by Hawks \&Teng, 1993[4].

A pavement is a kind of consumable material and must be replaced at an appropriate timing because it is easily and directly damaged or deteriorated by the load applied and surrounding environment, such as heavy traffic, intemperate climate and so on, depending on its location. Therefore, first, the causes of the degradation need to be investigated and explained for proper maintenance. Monitoring and assessment of the pavement condition should then be undertaken annually to ensure its efficiency for the preservation and management of road networks [5].

Pavement roughness is calculated using various automated multifunctional measuring instruments or tools and quantified using the International Roughness Index (IRI), an internationally agreed parameter

[6]. IRI was first used by the National Cooperative Highway Research Program in the late 70's and adopted as a universal scale by the World Bank [6]. IRI is measured by automation using a road profiler that produces a series of track profile numbers by combining reference elevation, reference height and longitudinal distance [7]. Response-type road roughness meters or profilers are typically used to collect IRI data and are usually mounted on computer-based specialized vehicles to monitor pavement roughness. The system tracks the displacement of the vehicle frame by unit distance traveled relative to the rear axle, typically in terms of counts per mile or foot [8]. As the larger IRI value is, the shake of vehicle body is also large, thus high IRI indicates uncomfortable riding [9]. The standard rating scale is according to Roughometer User Manual by ARRB, 2013[10], ranging from excellent to bad, which has also been adopted by of Roads, Nepal.

The pavement condition index (PCI) has been developed by the U.S. Army Corps of Engineers (1982). The PCI value is decreased by a cumulative deduct value score based upon the type, quantity, and severity level of distress and type of pavement [11]. Karan et al., 1983 provided a pavement quality index (PQI) approach for statistically collecting information from a panel of experts. It was developed from an analysis of 40 riding comfort index (RCI), structural adequacy index (SAI), and surface distress index (SDI) sections, each on a scale of 0 to 10[12]. Thube et al . (2007) developed composite pavement deterioration models based on PSI and PCI for low volume India roads.. Gharaibeh et al . ( 2010) compared pavement condition indices from five DOTs in the U.S. and the results showed significant differences between apparently similar pavement condition indices, which may be due to different types of distress considered, weighting factors and index mathematics, as the author concluded [12]. The PCI method is based on visual examination of the pavement distress type, extent and severity [13]. The PCI provides a measure of the current pavement condition based on the distress found on the pavement surface, which also indicates the structural integrity and operational state of the surface (roughness and safety). The standard rating scale ranges from excellent to failed as explained by Kirbas et al ,2010 [14].

There are different methods for collecting data on surface distress and these increase in complexity and sophistication depending on the amount of information needed. The department's method is a streamlined technique suggested by the World Bank, which has been updated to match the unique circumstances in Nepal and the need for DOR[15]. Pavement distress surveys are carried out manually 
in the Department by trained Highway Engineers working as a two person team; the method in use is a "drive and walk "survey. Surface distress comprises cracking, disintegration (potholes), deformation, texture deficiency, pavement edge defaults and maintenance work (patching). These faults are visually assessed using a $10 \%$ sampling procedure and recorded using a cumulative index called a surface distress index (SDI)[2].

Park et al. (2007) established a power relationship between PCI and IRI using data from nine states and provinces in Northern America. The IRI-PCI data set used in the study used were extracted from the DataPave program for the regions of Delaware, Maryland, New Jersey, New York, Vermont, Virginia, Ontario, Quebec and Prince Edward Island and spanned the period from 1991 through 2000. The power model proposed was which led to a transformed linear regression model as follows:

$$
\log P C I=2-0.436 \log (I R I)
$$

The $\mathrm{R}^{2}$ value of the model was determined to be $59 \%$. The plots of the residuals and normal scores were used to confirm the normality and homoscedasticity of the model's distribution[16].

In 2012, Shahnazri et al. (2012) estimated PCI values from other pavement indices (other than IRI) based on different types of distresses and severity levels using two optimization techniques: artificial neural networks (ANN) and genetic programming (GP). The models were developed based on PCI data gathered from more than $1,250 \mathrm{~km}$ of highways in Iran. A feed forward ANN was used with the network being trained using the back propagation method. In addition, the root-mean square error (RMSE) fitness function was used for the GP approach. From the results, the ANN- and GP-based projected values were determined to be in good agreement with the field-measured PCI values. The reported $\mathrm{R}^{2}$, RMSE and mean absolute error (MAE) for the ANN-based models were respectively $0.9986,0.99$, and 0.49 , whereas they were equal to $0.9898,2.63$, and 1.79 respectively for the GP-based model[17].

Another model for IRI as a function of PCI was developed for the Bay Area cities and counties in California with the intent of using the model in estimating user costs/benefits in their pavement management system. SPS statistical software was used for the modeling and regression validation resulting in the following equation:

$$
\mathrm{IRI}=0.0171(153-\mathrm{PCI})
$$

where, IRI is in $\mathrm{m} / \mathrm{km}$. The model's $\mathrm{R}^{2}$ value was 0.53 with a coefficient of variation of 28 percent. The actual and predicted values of IRI were compared graphically to depict the dispersion of data and for model [18].

In 2013, a neural network model was developed to estimate IRI from PCI. The model was however only based on data obtained for construction work zones. The predicted IRI values from the model were compared with the actual IRI values measured using MERLIN (Machine for Evaluating Roughness using Low-cost Instrumentation) along the construction work zones. The researchers used LevenbergMarquardt back-propagation for the estimation of IRI from PCI. The neural network model developed was trained and tested resulting in an $\mathrm{R}^{2}$ value of 0.86 and MSE of 0.041 which indicate that the performance of neural network is satisfactory and feasible for the prediction IRI [19].

Several relationships involving other pavement indices have been developed by several jurisdictions whose environmental conditions differ. In addition, some of these models may have been developed based on data compiled in suburban areas, and data from dense areas within that jurisdiction and may or may not have been included. As a result, a model developed for one jurisdiction is often inappropriate for another jurisdiction. In 2016, Suryoto and Siswoyo generated the correlation between the International Roughness Index and Surface Distress Index which is presented by the linear fit by the curve of $y=a+b x$ where $y=32.684+3.3455 x$, where $y$ is the value of SDI, $a=a$ constant value, $b=$ 
regression coefficient, and $\mathrm{x}$ is the value of IRI. Pearson correlation analysis of the curve indicates the value of $r=0.203$ based on the correlation coefficient table has a poor correlation. While the value of the standard error numbered of regression model to predict the value of y is 22.296 [20].

\section{Methodology}

This research work was conducted through the length of $23.6 \mathrm{Km}$ on different feeder roads emerging outside of ringroad of Kathmandu and Lalitpur districts. Each kilometer was divided on 10 samples each of $100 \mathrm{~m}$. This study focuses on estimation of road pavement condition through manual surveys using the Pavement Condition Index (PCI) and Surface Distress Index (SDI). PCI being the best index to reflect the cause of deterioration, but is not in practice in Nepal due to cumbersome process of its calculation. The pavement existing condition was evaluated in reference to SDI, IRI and PCI for the pavement for which survey was conducted. Other data required was collected from the Department of Roads, Highway Management and Information System (HMIS). This research aims to develop a relationship between road roughness and surface distress indices. This relation would reduce the labor hour for collection of PCI data which ultimately will save the cost of surveying conducted by DOR. The outcome of this research may reduce the time for collecting, reviewing and processing distress photographs for PCI determination and thereby eliminating the annual routine subjective rating of pavement distress. In addition, the expense for obtaining and analyzing the field data for determining the PCI could be eliminated, except for specific purpose of upgrading established relationships. PCI for measuring the surface distress has advantage over SDI for measuring the same. The range of categorization is wide according to PCI than that of SDI. More to say, PCI has range starting from zero to hundred, whereas, SDI has minimal range from zero to five.

\section{Data Analysis}

\section{PCI-Analysis}

Distress types, severity levels and density were determined. The total quantity of each distress type at each severity level were added, and recorded in the" Total severities" section. The unit for the quantities may be either in square feet (square meter), linear feet (meters), or number of occurrences, depending the type of distress. The total quantity of each distress type at each severity level were divided by the total area of the sample unit and multiplied by 100 to obtain the percent of each distress type and severity. The deduct value (DV) were determined for each distress deduct value curves in ASTM standard D 6433. The maximum corrected deduct value (CDV) were determined. The procedure were used to determine the maximum CDV and steps as mentioned in ASTM standard D 6433[21].

\section{SDI-Analysis}

The full width of the pavement was examined for the 100 metre sample length. The survey was carried out through the whole portion of the pavement dividing it to equal 100 meter samples. The distress was then recorded on the survey sheet in considering the sample units and size according to the severity. While walking, it was necessary to first identify the major and minor defects and then calculate the percentage of the total defective area for both major and minor defects individually. The collected data from the field was then used for the further analysis and scoring of pavement in consideration of SDI. The respective SDI for each sample unit is the calculated in an excel sheet.

\section{IRI-Analysis}

The raw data were obtained from the HMIS section. These data were then processed to achieve the desired objective of the study area. These data were then computed using software known as Roughometer software. This software is compatible with the Roughometer device used for collecting IRI data in the field. Once a survey has been undertaken, the Roughometer III processing software enables the data to be formatted into custom graphs, tables and maps. 


\section{$5 \quad$ Findings}

The relationship has been developed relating SDI, PCI with IRI. In 2017, out of $23.6 \mathrm{Km}$ taken for the study, data of $18.9 \mathrm{Km}$ were taken for developing the relationships between pavement roughness and distress. The remaining lengths were kept for validating the relationships. Moreover, the developed relationships were validated with the latest data of 2019 . The latest data of $3 \mathrm{Km}$ for SatdobatoGodavari was taken for validation.

\subsection{Relationship between PCI and IRI}

The relationship between PCI and IRI can be developed in various form as of Linear, exponential, and polynomial functions.

\section{Polynomial Relationship between PCI and IRI}

The Polynomial Relationship between PCI and IRI was obtained from the partial samples obtained during the study. The relation was obtained from the data plotted on the polynomial scale graph as shown on figure 1 which can be formulated as:

$$
\mathrm{PCI}=0.1003 \times \mathrm{IRI}^{2}-7.944 \times \mathrm{IRI}+100.62
$$

Considering the $\mathrm{R}^{2}$ value of the three relationships developed for PCI and IRI, the polynomial equation with power two can be considered the best fit relationship.

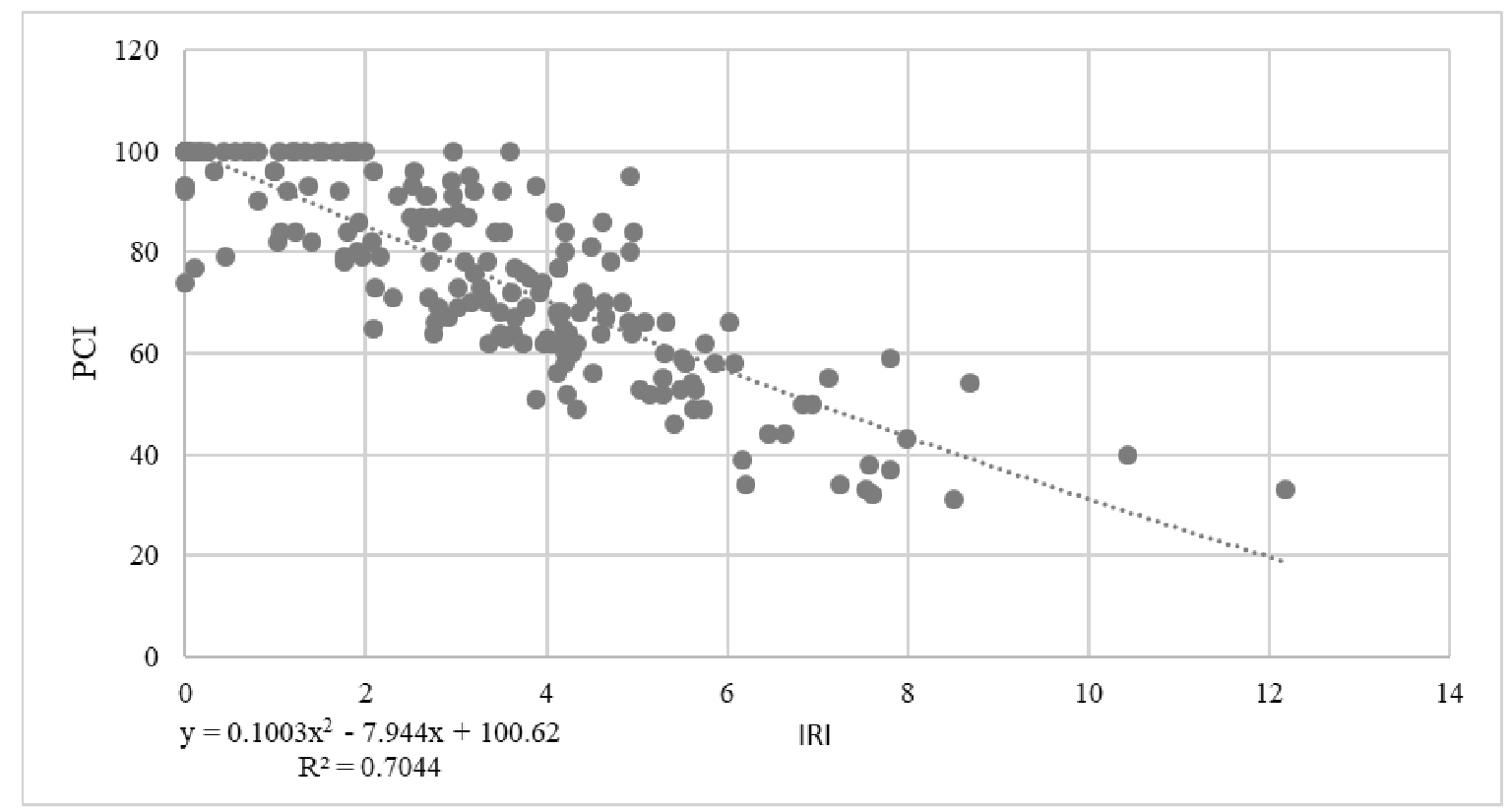

Fig 1 Polynomial Relationship between PCI \& IRI

\section{Linear Relationship between PCI and IRI}

The Linear Relationship between PCI and IRI was obtained from the partial samples obtained during the study. The relation was obtained from the data plotted on the linear scale graph which can be formulated as:

$$
\mathrm{PCI}=-7.14 \mathrm{IRI}+99.51
$$

The negative denotation in the linear relationship between PCI and IRI was due to the categorization schemes for PCI and IRI is opposite. 


\section{Exponential Relationship between PCI and IRI}

The Exponential Relationship between PCI and IRI was obtained from the partial samples obtained during the study. The relation was obtained from the data plotted on the exponential scale graph which can be formulated as:

$$
\mathrm{PCI}=104.38 \mathrm{e}^{-0.107(\mathrm{IRI})}
$$

\section{Logarithmic Relationship between PCI and IRI}

The Logarithmic Relationship between PCI and IRI could not be obtained from the partial samples obtained during the study. It is due to the nature of categorization of IRI and PCI.

\subsection{Relationship between SDI and IRI}

The relationship between SDI and IRI can be developed in various form as of Linear and polynomial functions.

\section{Polynomial Relationship between SDI and IRI}

The Polynomial Relationship between SDI and IRI was obtained from the partial samples obtained during the study. The relation was obtained from the data plotted on the polynomial scale graph as shown on figure 2 which can be formulated as:

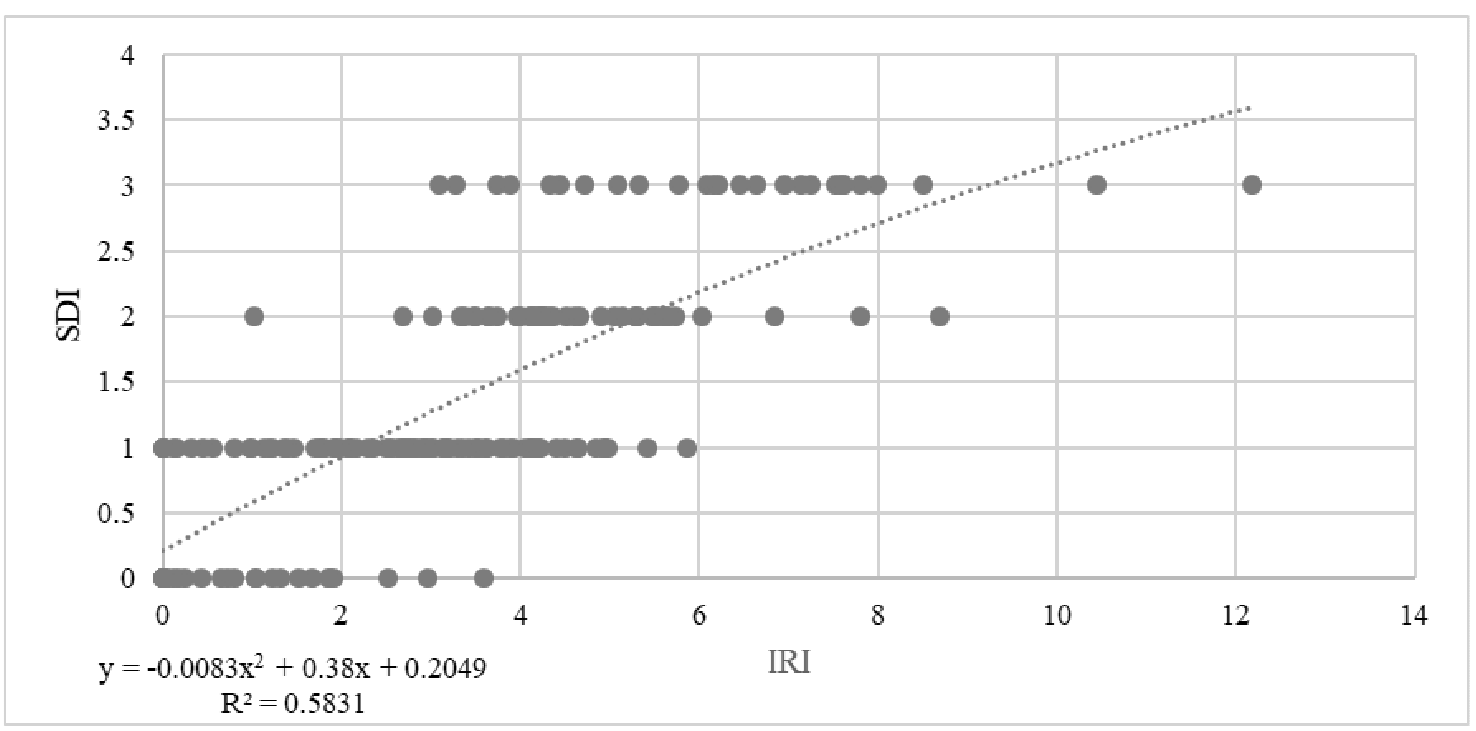

Fig 2 Polynomial Relationship between SDI \& IRI

$$
\mathrm{SDI}=-0.0083 \mathrm{IRI}^{2}+0.38 \mathrm{x} \mathrm{IRI}+0.2049
$$

Considering the $\mathrm{R}^{2}$ value of the two relationships developed for SDI and IRI, the polynomial equation with power two can be considered the best fit relationship. Due to the nature of data of IRI and SDI, the exponential relationship for this two indices could not be obtained.

\section{Linear Relationship between SDI and IRI}

The Linear Relationship between SDI and IRI was obtained from the partial samples obtained during the study. The relation was obtained from the data plotted on the linear scale graph which can be formulated as:

$$
\mathrm{SDI}=0.3127 \mathrm{IRI}+0.2971
$$

The positive denotation in the linear relationship between SDI and IRI was due to the categorization schemes for PCI and IRI is similar. 


\section{Exponential and Logarithmic Relationship between SDI and IRI}

Due to the nature of data of IRI and SDI, the exponential and logarithmic relationship for this two indices could not be obtained.

\section{Validations}

The R2 value for PCI- IRI relationship was 0.70 , which indicates $70 \%$ of the data has been fitted with the polynomial relationship developed. Also, adjusted $\mathrm{R} 2$ value is found to be 0.70 . In addition to this, p-value (t-test) for the F-statistic for regression determined to be less than 0.05 that is nearly to 0 indicating that the relationship between PCI and IRI is quite adequate. The calculated chi-square value was found to be 47.0 . For $5 \%$ level of significance and degree of freedom as 46 , the critical chi-square value is 62.83 . Since the critical chi-square value was greater than calculated, it shows the relationship can be used for PCI prediction.

In addition to this, the relationship was validated with the latest data of 2019 for Satdobato-Godavari Section. IRI was obtained from the HMIS unit. PCI was calculated by carrying out the survey. The calculated chi-square value was found to be 37.7. For $5 \%$ level of significance and degree of freedom as 29 , the critical chi-square value is 42.5 . Since the critical chi-square value is greater than calculated, it shows the relationship can be used for PCI prediction.

The R2 value for SDI- IRI relationship was 0.58 , which indicates $58 \%$ of the data has been fitted with the polynomial relationship developed. Also, adjusted $\mathrm{R} 2$ value is found to be 0.58 . In addition to this, p-value (t-test) for the F-statistic for regression determined to be less than 0.05 that is nearly to 0 indicating that the relationship between SDI and IRI is quite adequate. The calculated chi-square value was found to be 9.1 . For $5 \%$ level of significance and degree of freedom as 46 , the critical chi-square value is 62.83 . Since the critical chi-square value was greater than calculated, it shows the relationship can be used for SDI prediction.

In addition to this, the relationship was validated with the latest data of 2019 for Satdobato-Godavari Section. IRI was obtained from the HMIS unit. SDI was calculated by carrying out the survey. The calculated chi-square value was found to be 3.1. For 5\% level of significance and degree of freedom as 29 , the critical chi-square value is 42.5 . Since the critical chi-square value is greater than calculated, it shows the relationship can be used for SDI prediction.

\section{Conclusions}

The relationships were developed among the indices in the study. Pavement roughness index, IRI was compared and related to Pavement distress indices, SDI and PCI. Among the various relationships developed between PCI and IRI, the polynomial relationship was considered to be best fit with $\mathrm{R}^{2}$ value as 0.70 . Moreover, among the multiple relationships between SDI and IRI, polynomial relationship was found to be best with $\mathrm{R}^{2}$ value of 0.58 . This showed the relationship of PCI with IRI shows good fit rather than that with SDI. Due to limitations in pavement evaluation categorizations considering SDI, and variance of categorizations in PCI the fit in relation for PCI is countable.

Furthermore, the study was done to find the relationship between surface distress indices, PCI and SDI. Among various relationships developed, polynomial relationship was found to be best fit, with $\mathrm{R}^{2}$ value of 0.66 . The $\mathrm{R}^{2}$ value for SDI and PCI was found quite low than relationship between PCI-IRI. It must be due to wide variation in rating scales of SDI and IRI.

The different relationships obtained among the indices was found to have good output while tested using F-test and p-test, along with chi-square test with 5\% level of significance. The F-value for PCIIRI relationship was found to be 442.15 and p-value as nearly about to zero. Similarly, for SDI-IRI relationship was found to be 256.39 and p-value as nearly about to zero. Furthermore all the 
relationships developed were validated using the latest data of 2019, which satisfied the chi-square test for accepting the polynomial relationship between each of the two indices.

\section{Recommendations}

Based on the findings of the research, following recommendations are drawn for pavement evaluation. The Government of Nepal uses Surface Distress Index to measure the distress and categorize the road surface. As, PCI is found more reliable and descriptive with variation in categorizing schemes, using of PCI for road evaluation is recommendable in compared to SDI and IRI. The life span of pavement can be enhanced by properly evaluating the pavement condition by PCI which could not be achieved by other evaluating indices, this has become easy by use of the relationship developed from the study.

\section{Acknowledgements}

My gratitude to director of Nepal Engineering College - Center for Postgraduate Studies (nec-CPS) Prof. Dr. Khem Raj Sharma, Research Coordinator, Dr. Anish Ghimire, Dr. Thusitha Chandani Shahi (Transportation Engineering and Management, nec-CPS), for their guidance and support for the completion of this study. I would also like to thank all faculty members and teachers of Nepal Engineering College, Pokhara University for their valuable supports and guidance during the entire study period.

\section{References}

1. Adlinge, S. S. \& Gupta, A. K., 2015. "Pavement Deterioration and its Causes". Journal of Mechanical \& Civil Engineering, pp. 2778-1684.

2. DOR, 1995. "Road Pavement Managent Discussion Paper”, s.1.: Department of Roads.

3. Kwaku, O.-A., 2016. "A Study on factors that contribute to pavement deteriroration on the Mampong Arterial (Suame Roundabout-Pankrono Road)”, Kabwe,Zambia: s.n.

4. Hawks, N. E. \& Teng, T. P., 1993. "Distress Identification Manual for the Long-Term Pavement Performance Projecct”, Washington, DC: National Academy of Sciences.

5. Salpisoth , H., 2014. "Simple evaluation methods for road pavement management in developing country”, s.1.: Kyoto University.

6. Arhin, S. A. \& Noel, E. C., 2014. "Predicting Pavement Condition Index Using International Roughness Index in Washington DC", Washington DC: District Department of Transportation Research, Development, \& Technology Transfer Program.

7. Sayers, M. W., Gillespie, T. D. \& Paterson, W. D., 1986. "Guidelines for Conducting and Calibrating Road Roughness Measurements”, Washington, D.C., U.S.A.: s.n.

8. Shafizadeh, K., Mannering, F. \& Pierce, L., 2002. “A Statistical Analysis of Factors Associated with Driver-Perceived Road Roughness on Urban Highways", Washington DC: Washington State Transportation Commission.

9. Nagayama, T., Miyajima, A., Kimura, S., Shimada, Y. and Fujino, Y., 2013, April. "Road condition evaluation using the vibration response of ordinary vehicles and synchronously recorded movies." In Sensors and Smart Structures Technologies for Civil, Mechanical, and Aerospace Systems 2013 (Vol. 8692, p. 86923A). International Society for Optics and Photonics.

10. ARRB, 2013. “User Manual Roughometer III.” UM-RM3-USR ed. s.1.:ARRB Group Ltd.

11. Wu, K., 2015. "Development of PCI-based Pavement Performance Model for Management of Road Infrastructure System”, s.1.: s.n. 
12. Shah, Y. U., Jain, S. S., Tiwari, D. \& Jain, M. K., 2013. "Development of Overall Pavement Condition Index for Urban Road Network." s.l., Elsevier.

13. ASTM, 2007. ASTM D 6433-07, "Standard Practice for Roads and Parking Lots Pavement Condition Index Surveys”, s.1.: s.n.

14. Kirbas, U. and Gursoy, M., 2010. "Developing the basics of pavement management system in Besiktas district and evaluation of the selected sections." Scientific Research and Essays, 5(8), pp.806-812.

15. DOR, 2015. "Draft Report on Traffic, Surface Distress and Road Roughness Surveys on SRN", Kathmandu: DOR/HMIS UNIT.

16. Park, K., Thomas, N. E. \& Lee, K. W., 2007. “Applicability of the International Roughness Index as a Predictor of Asphalt Pavement Condition. Journal of Transport Engineering”, 133(12), pp. 706-709.

17. Shahnazri, H., Tutunchain, M. A., Mashayekhi, M. \& Amini, A. A., 2012. “Application of Soft Computing for Prediction of Pavement Condition Index.” Journal of Transportation Engineering, 138(12).

18. Arhin, S. A., Williams, L. N., Ribbiso, A. \& Anderson, M. F., 2015. "Predicting Pavement Condition Index Using International Roughness Index in a Dense Urban Area. ” Journal of Civil Engineering Research, 5(1), pp. 10-17.

19. Vidya, R., Mathew, S. \& Santhakumar, S. M., 2013. "Estimation of IRI from PCI in Construction Work Zones. ” Int. J. on Transportation and Urban Development, 3(1).

20. Suryoto, A. S. \& Siswoyo, D. P., 2016. "The evaluation of functional performance of national roadway using three types of pavement assessments methods. Central Java", Procedia Engineering, pp. 1435-1442.

21. ASTM, 2007. ASTM D 6433-07, "Standard Practice for Roads and Parking Lots Pavement Condition Index Surveys”, s.1.: s.n. 